\title{
INFORMAÇÃO COMO INSTRUMENTO DA ASSISTÊNCIA AO PACIENTE SUBMETIDO A CIRURGIA ORTOPÉDICA
}

\author{
Isabel Cristina Oliveira Vital ${ }^{1}$, Lys Eiras Cameron ${ }^{2}$, Thays Regina da Cunha ${ }^{3}$, Caroline Intorne Santos ${ }^{4}$
}

\begin{abstract}
RESUMO: Objetivo: identificar as informações recebidas pelos pacientes, relacionadas aos períodos perioperatório e alta hospitalar de Artroplastia Total de Quadril. Método: pesquisa observacional, tipo survey, realizada em três hospitais públicos no Rio de Janeiro em 2010, 2011, 2013 e 2015. Dados coletados com 113 indivíduos hospitalizados com indicação de cirurgia ortopédica, por entrevista, organizados em um quadro categorizando as informações pelos períodos perioperatórios e alta, e analisados utilizando estatística descritiva simples. Resultados: predominaram mulheres, idosos e a principal indicação foi fratura. Dezesseis informações foram mencionadas pelos pacientes: oito sobre pré-operatório, uma sobre transoperatório e sete sobre pósoperatório das quais somente quatro estavam relacionadas ao período após a alta. Conclusão: os resultados demonstram a insuficiência de informações sobre o perioperatório de cirurgia ortopédica para atender às necessidades dos pacientes e que o conhecimento deficiente do paciente e seu cuidador pode levar a complicações evitáveis e causar deterioração do estado de saúde.
\end{abstract}

DESCRITORES: Informação; Assistência ao Paciente; Enfermagem; Relações Profissional-Paciente; Enfermagem Ortopédica.

\section{INFORMATION AS AN INSTRUMENT OF CARE TO PATIENTS UNDERGOING ORTHOPEDIC SURGERY}

ABSTRACT: Objective: to identify the information obtained from patients who underwent Total Hip Arthroplasty (THA), related to the perioperative periods and after hospital discharge. Method: Observational study, of survey-type, conducted in three public hospitals in Rio de Janeiro in 2010, 2011, 2013 and 2015. Data collected through interviews with 113 patients admitted to hospital with indication for orthopedic surgery was organized into a table where the information was classified by perioperative periods and after discharge, and analyzed using simple descriptive statistics. Results: Prevalence of women, elderly and the main indication was fracture. Sixteen pieces of information were mentioned by the patients: eight on the preoperative period, one in the intraoperative period and seven on the postoperative period, of which only four were related to the post discharge period. Conclusion: According to the results, there is scarce information about the perioperative period of orthopedic surgery to meet the needs of patients and the poor knowledge of patients and caregivers about this period may lead to avoidable complications and cause deterioration of health status.

DESCRIPTORS: Information; Patient Care; Nursing; Professional-Patient Relationship; Orthopedic Nursing.

\section{INFORMACIÓN COMO INSTRUMENTO DE LA ASISTENCIA AL PACIENTE SOMETIDO A CIRUGÍA ORTOPÉDICA}

RESUMEN: Objetivo: identificar informaciones acerca de los periodos perioperatorio y alta hospitalaria de Artroplastia Total de Cuadril provenientes de los pacientes. Método: investigación observacional, del tipo survey, realizada en tres hospitales públicos en Rio de Janeiro en 2010, 2011, 2013 y 2015. Se obtuvieron los datos de 113 individuos hospitalizados con indicación de cirugía ortopédica, por medio de entrevista, organizados en un cuadro de informaciones por categorías de periodos perioperatorios y alta, y que fueron analizados utilizándose estadística descriptiva simple. Resultados: hubo predominancia de mujeres, ancianos y la principal indicación fue por fractura. Los pacientes mencionaron dieciséis informaciones: ocho acerca del preoperatorio, una sobre transoperatorio y siete sobre posoperatorio. De estas solamente cuatro estaban relacionadas al periodo después del alta. Conclusión: los resultados muestran la insuficiencia de informaciones sobre el perioperatorio de cirugía ortopédica para atender a las necesidades de los pacientes y que el conocimiento deficiente del paciente y de su cuidador pueden causar complicaciones evitables, además de empeorar el estado de salud.

DESCRIPTORES: Información; Asistencia al Paciente; Enfermería; Relaciones Profesional-Paciente; Enfermería Ortopédica.

${ }^{1}$ Enfermeira. Mestre em Enfermagem. Consultora de Serviços de Saúde da Empresa DBV. Rio de Janeiro, RJ, Brasil.

${ }^{2}$ Enfermeira. Doutora em Enfermagem. Docente de Enfermagem da Universidade Federal do Rio de Janeiro. Rio de Janeiro, RJ, Brasil.

${ }^{3}$ Enfermeira. Mestre em Enfermagem. Enfermeira Educadora da UHG Brasil. Rio de Janeiro, RJ, Brasil.

${ }^{4}$ Enfermeira. Mestranda em Enfermagem. Enfermeira da Fundação de Saúde do Estado do Rio de Janeiro. Rio de Janeiro, RJ, Brasil. 


\section{INTRODUÇÃO}

A Artroplastia Total de Quadril é uma cirurgia de substituição da articulação do quadril, indicada para doenças degenerativas articulares e fraturas nessa região, proporcionando alívio da dor, recuperação da mobilidade articular e melhora sensível da qualidade de vida. O ato cirúrgico envolve responsabilidades do hospital, da equipe de saúde, do paciente e familiares em diversos graus, e para resultados pósoperatórios positivos, o adequado preparo do paciente tem fundamental importância ${ }^{(1-5)}$.

Qualquer cirurgia, seja eletiva ou de emergência, é um procedimento complexo. Apesar do ambiente e das rotinas de cuidados relacionados ao tratamento variarem entre as instituições, após a indicação da cirurgia é fundamental um cuidadoso planejamento. Por ser uma das cirurgias mais complexas de Ortopedia, demanda da Enfermagem e demais profissionais da equipe de saúde um grande conhecimento técnico e científico para a assistência, não só durante a hospitalização, mas também para o preparo do paciente e seus familiares para a alta e reabilitação.

A possibilidade de ocorrência de complicações nos pacientes cirúrgicos ortopédicos chama a atenção para a importância das intervenções adequadas durante a hospitalização, um acompanhamento criterioso e preparo para a alta. As informações fornecidas ao paciente sobre o pré, trans e pósoperatório, não só pela Enfermagem mas por toda equipe que o assiste, tem especial impacto na recuperação da saúde desse grupo de pacientes ${ }^{(2,4-5)}$.

De acordo com DeCS - Descritores em Ciências da Saúde, informação pode ser definida como a comunicação ou recepção de conhecimentos sobre determinado assunto. Nesse contexto, entendendo informação como o meio pelo qual o indivíduo toma conhecimento e tem poder de determinação sobre a sua condição de saúde e tratamento, neste estudo utilizou-se o termo "informação" para a comunicação ou recepção de um ou de um conjunto de conhecimentos sobre determinado assunto, seja na forma de orientação, intervenções educacionais, aconselhamento, instrução, treinamento, ensino, educação para a saúde ou qualquer outra atividade semelhante realizada com o paciente e seu familiar ou cuidador.

A informação ao paciente, centrada nas suas necessidades individuais a partir de protocolos estabelecidos pela instituição de saúde, diminui o nível de ansiedade e o medo relacionado ao préoperatório e cirurgia e aumenta o entendimento sobre o processo de tratamento, garantindo a qualidade dos cuidados e o resultado final positivo. Dá ao paciente e sua família condições de lidar com seus problemas de saúde e possibilita antecipar o que deseja conhecer. Deve enfatizar a importância para o paciente gerenciar seus problemas de saúde, estimulando a sua autonomia, levando em consideração a sua capacidade de compreensão, motivações, conhecimento prévio e experiências ${ }^{(6-8)}$.

Segundo dados de janeiro de 2017 do DATASUS ${ }^{(9)}$, de um total de 438.690 leitos disponíveis no Brasil para internações, 17.488 são dedicados aos pacientes cirúrgicos ortopédicos, mais que as internações clínicas e cirúrgicas da Cardiologia, por exemplo (12.974 leitos). Associa-se a isto uma reduzida produção de artigos em periódicos nacionais assim como de livros de Enfermagem Traumato-Ortopédica, além da dificuldade para o acesso a periódicos internacionais sobre a especialidade. Nesse contexto, há uma evidente necessidade de estudos para desenvolver pesquisas nessa área da Enfermagem e, em especial, sobre a educação do paciente submetido à cirurgia ortopédica, de forma que a equipe seja capaz de fornecer informações no momento certo e da maneira correta, atendendo à real necessidade do paciente.

Desta forma, este artigo se justifica por refletir sobre a identificação das necessidades de informação sobre o período de hospitalização e após a alta de pacientes de cirurgias ortopédicas em pacientes submetidos à Artroplastia Total de Quadril. Esses princípios de necessidade de oferecer a informação e seu reforço durante todo o período de tratamento podem ser aplicados a pacientes de qualquer clínica cirúrgica. Nesse contexto, o objetivo deste estudo é identificar as informações recebidas pelos pacientes, relacionadas aos períodos pré, trans, pós-operatório e alta hospitalar de Artroplastia Total de Quadril.

\section{- MÉTODO}


Trata-se de pesquisa de abordagem quantitativa, observacional, do tipo survey. Os dados foram coletados com 113 pacientes hospitalizados. Foram incluídos indivíduos de ambos os sexos, com idade superior a 18 anos, submetidos a Artroplastia Total de Quadril, internados em três hospitais públicos na cidade do Rio de Janeiro. Foram excluídos do estudo os indivíduos não preservados cognitivamente.

Os dados foram coletados em três períodos diferentes: de outubro de 2010 a outubro de 2011, maio a julho de 2013 e abril de 2015, de segunda a sexta, em horário previamente combinado com os participantes. Os dados relacionados às informações relacionadas ao pré, trans, pós-operatório e alta de Artroplastia Total de Quadril, lembradas como fornecidas aos pacientes para os diferentes períodos de tratamento, foram coletados pelos pesquisadores por entrevista e organizadas em um quadro categorizando as informações por períodos: pré, trans, pós-operatório (durante a hospitalização e após a alta). Após foram registrados no Programa Microsoft Excel e submetidos à estatística descritiva.

Para a discussão dos resultados, a identificação das informações necessárias ao paciente submetido à cirurgia em questão foi construída a partir de uma busca nos seguintes bancos e bases de dados: Base de dados de enfermagem (BDENF); Biblioteca Cochrane; Cummulative Index to Nursing and Allied Health Literature (CINAHL); EBSCO Information Services; Excerpta Medica dataBASE (EMBASE); Literatura Latino-Americana e do Caribe em Ciências da Saúde (LILACS); PubMed; Scientific Electronic Library Online (SciELO) e SciVerse Scopus (SCOPUS). Foram incluídos na seleção os artigos publicados nos últimos 10 anos (2006-2016) em português, inglês e espanhol, disponíveis em texto completo. Os descritores selecionados em MeSH ${ }^{\otimes}$ foram: "arthroplasty, replacement, hip" AND "Total Hip Replacement" e "Artroplastia de Quadril" em DeCS.

Este projeto foi submetido à apreciação dos Comitês de Ética em Pesquisa das instituições escolhidas (Pareceres 312A.2010; 36.10; 2720), de acordo com o estabelecido pela Resolução 466/2012 ${ }^{(10)}$.

\section{- RESULTADOS}

Dos 113 pacientes que participaram desta pesquisa, 71 eram mulheres $(62,8 \%)$ e $42(37,2 \%)$ homens. As idades variaram de 29 a 92 anos (média 76,6 6, 6,34), sendo predominante a faixa etária entre 60 e 89 anos (87 indivíduos; 77\%). As indicações para a realização da Artroplastia Total de Quadril foram: fratura (54 indivíduos; 47,8\%), osteoartrite (20;17,7\%), necrose avascular (19; 16,8\%), infecção (11; 9,7\%) e revisão cirúrgica (nove; 8\%). As queixas apresentadas pelos participantes desta pesquisa durante o pré-operatório, relacionadas à causa de indicação para a cirurgia, foram: dor (97; 85,8\%), limitação funcional $(96 ; 84,9 \%)$, restrição às atividades cotidianas $(91 ; 80,5 \%)$, rigidez $(38 ; 33,6 \%)$ e formigamento (dois; $1,8 \%$ ).

Este estudo elencou as informações apontadas pelos pacientes, lembradas como recebidas e relacionadas aos cuidados no período pré, intra e pós-operatório de Artroplastia Total de Quadril, fornecidas por qualquer profissional da saúde que tenha tido participação na assistência. Todas essas informações foram reconhecidas pelos pacientes como importantes para o seu tratamento.

As informações apontadas pelos 113 pacientes como fornecidas durante o período de internação hospitalar nos três hospitais pesquisados estão apresentadas no Quadro 1.

Quadro 1 - Informações apontadas pelos 113 participantes submetidos à Artroplastia Total de Quadril. Rio de Janeiro, RJ, Brasil, 2015 (continua)

\begin{tabular}{|l|c|c|}
\hline Informação & $\mathbf{n}(\mathbf{\%})$ & Período \\
\hline Indicação da cirurgia & $109(96,4)$ & Pré-operatório \\
\hline Higiene corporal pré-operatória & $81(71,7)$ & Pré-operatório \\
\hline Necessidade de manutenção do jejum pré-operatório & $41(36,2)$ & Pré-operatório \\
\hline Local e o tipo de anestesia & $28(24,8)$ & Trans-operatório \\
\hline Cuidados com o curativo e dreno & $28(24,8)$ & Pós-operatório (hospital) \\
\hline Acompanhamento fisioterápico após a alta hospitalar & $21(18,6)$ & Pós-operatório (alta) \\
\hline
\end{tabular}




\begin{tabular}{|l|c|c|}
\hline Realização de exercícios ao leito no pós-operatório hospitalar & $19(16,8)$ & Pós-operatório (hospital) \\
\hline Necessidade de avaliação de outros especialistas antes da cirurgia & $18(15,9)$ & Pré-operatório \\
\hline Manutenção da almofada de dupla abdução & $18(15,9)$ & Pós-operatório (hospital) \\
\hline Uso de medicamento para a dor após a alta & $17(15)$ & Pós-operatório (alta) \\
\hline Avaliação pré-operatória do anestesista & $10(8,8)$ & Pré-operatório \\
\hline Retirada de prótese dentária antes da cirurgia & $10(8,8)$ & Pré-operatório \\
\hline Realização de curativo após a alta & $6(5,3)$ & Pós-operatório (alta) \\
\hline Necessidade de suspensão da cirurgia & $6(5,3)$ & Pré-operatório \\
\hline Mobilização ao leito no pré-operatório & $3(2,6)$ & Pré-operatório \\
\hline Tempo de reabilitação pós-operatória & $1(0,9)$ & Pós-operatório (alta) \\
\hline
\end{tabular}

É importante ressaltar que, apesar de ser conhecimento dos pesquisadores que nos três hospitais pesquisados existem rotinas estabelecidas para os cuidados perioperatórios dos pacientes em todas as equipes, nenhuma outra informação foi mencionada pelos indivíduos pesquisados além dessas registradas aqui, fossem relacionadas ao período de hospitalização ou para a alta hospitalar.

\section{- DISCUSSÃO}

Novas técnicas cirúrgicas combinadas com permanências hospitalares mais curtas reduzem a possibilidade de adequado e individualizado preparo do paciente para tratamento e reabilitação. Desta forma, o início das intervenções educacionais deve acontecer o mais precocemente possível, de forma a garantir tempo suficiente para que o paciente compreenda e retenha melhor as informações recebidas, sem as quais pode estar inadequadamente preparado para lidar com os problemas que surgem durante hospitalização e em casa, aumentando o risco de complicações e mortalidade ${ }^{(7)}$.

Neste estudo, apresentamos os dados relativos a 113 pacientes submetidos à Artroplastia Total de Quadril, uma das cirurgias mais complexas na Ortopedia. A assistência de Enfermagem a esses pacientes demanda conhecimento para todas as etapas perioperatórias e após a alta hospitalar, de tal forma que o paciente evolua positivamente no tratamento proposto e as complicações possam ser evitadas ou reduzidas.

Os dados desta pesquisa estão de acordo com a literatura que aponta que as Artroplastias de Quadril são mais frequentes em mulheres que em homens, assim como predominam entre os idosos $^{(3,8)}$. O objetivo do tratamento de idosos submetidos à Artroplastia Total de Quadril é restaurar a independência, a mobilidade, garantir um bom estado mental e a redução do risco da mortalidade prematura. Quando é realizada em indivíduos mais jovens, a preocupação se situa na durabilidade e sobrevivência dos implantes e no acompanhamento das complicações tais como o afrouxamento, desgaste e deslocamento ${ }^{(11)}$.

Fratura, osteoartrite e necrose avascular foram as principais causas de indicação cirúrgica entre os pesquisados, sendo predominantes as fraturas. As demais indicações foram infecção e revisão cirúrgica. A maioria das fraturas de quadril são causadas por acidentes e são uma das principais causas de morbidade e a mortalidade entre os idosos. A osteoartrite se destaca como uma das doenças articulares mais comuns na população idosa e é uma das principais causas de morbidade e incapacidade nesse grupo. A revisão operatória geralmente está relacionada à falha dos implantes, que pode ocorrer por razões mecânicas (tempo prolongado de uso da prótese, luxação, fratura óssea periprótese) ou biológicas (inflamação, infecção) $)^{(1,6,11-12)}$.

Dor, limitação funcional e restrição às atividades cotidianas estavam presentes em mais da metade dos pacientes, seguidas por rigidez e formigamento. Estes sintomas estabelecem um padrão reconhecido por relação direta com as indicações cirúrgicas apresentadas neste estudo e, nesses casos, a Artroplastia Total de Quadril geralmente apresenta resultados satisfatórios para a resolução desses problemas ${ }^{(6)}$. 
Os 113 pacientes hospitalizados em três instituições diferentes que participaram desta pesquisa citaram somente 16 informações: oito relacionadas ao pré-operatório, uma sobre o trans-operatório e sete sobre o pós-operatório sendo que, destas, somente três estavam relacionadas ao período após a alta.

Com relação ao período pré-operatório, a prática demonstra que pacientes adequadamente informados sobre o tratamento e com reforço dessas informações durante a hospitalização tendem a evoluir de forma diferenciada, com rápido restabelecimento e melhores resultados futuros. Além disso, todos os procedimentos realizados devem ser explicados de maneira que o paciente seja estimulado a participar ativamente do plano terapêutico proposto, compreendendo suas limitações e possibilidades. Essas informações devem incluir o período de hospitalização, transcender os muros da instituição de saúde e contemplar seu dia a dia, de forma a minimizar as complicações que podem estar relacionadas ao pós-operatório ${ }^{(7)}$.

As informações citadas pelos indivíduos pesquisados e relacionados ao pré-operatório, apesar de importantes, não contemplam em sua totalidade as informações mínimas para o paciente evoluir satisfatoriamente nesse período.

Várias informações fundamentais relacionadas ao período pré-operatório não foram citadas pelos indivíduos pesquisados, tais como: informações sobre tipo e tempo de manutenção da prótese; risco da cirurgia e possíveis complicações; restrições e limitações relacionadas à cirurgia; consentimento para a cirurgia; problemas e intervenções relacionados com ansiedade, medo, distúrbios do sono; estímulo ao autocuidado dentro das limitações do paciente; cuidados gerais de Enfermagem e de prevenção da síndrome do desuso; medicamentos específicos para problemas identificados e alterações de esquemas terapêuticos cotidianos; realização de exames laboratoriais e por imagem; avaliação préoperatória pelo enfermeiro do Centro Cirúrgico ${ }^{(6,13-20)}$.

Além desses, também foram omitidos: riscos relacionados à anestesia; informações sobre o protocolo de cirurgia segura; necessidade de suporte social; cuidados e restrições durante a recuperação pósanestésica e após o retorno à unidade de internação; limitações no pós-operatório hospitalar e após a alta; cuidados após a alta; adaptações e modificações da residência; presença e controle da dor pré e pós-operatória; necessidade de transfusão de sangue; evolução e tratamento das comorbidades; necessidade de ser encaminhado à Unidade de Terapia Intensiva no pós-operatório; e, medicamentos pré-anestésicos e encaminhamento ao Centro Cirúrgico ${ }^{(6,13-20)}$.

Com relação às informações apontadas pelos pacientes relacionadas ao período trans-operatório, estavam somente aquelas diziam respeito ao local e o tipo de anestesia, mencionadas por reduzido número de indivíduos que participaram deste estudo.

Demais informações relacionadas ao período em que permaneceriam no Centro Cirúrgico, imediatamente antes e após a cirurgia, não foram mencionadas, tais como: recepção e avaliação do paciente pelo enfermeiro e pelo anestesista; encaminhamento à sala de cirurgia e posicionamento para a anestesia; posicionamento seguro para a cirurgia; checklist de cirurgia segura; necessidade de medicações e transfusão sanguínea; utilização de almofada de dupla abdução (triângulo abdutor ou travesseiro abdutor) logo após a cirurgia para evitar a luxação da prótese $\mathrm{s}^{(3-4,6-6,71,20-21)}$.

Da mesma forma, foram omitidas informações sobre restrição absoluta ao leito; transferência para a recuperação pós-anestésica; presença de sondas, drenos, cateteres e curativo cirúrgico; monitorização; vigilância e segurança pós-operatória; controle da dor; medidas específicas para controle de náuseas, vômitos, confusão, agitação; hipotermia; controle da situação hemodinâmica; profilaxia de trombose venosa profunda; informações sobre o intraoperatório; controle de situações de risco; encaminhamento à unidade de terapia intensiva $a^{(3-4,6-6,74,20-21)}$.

Embora existam informações relacionadas aos cuidados que habitualmente a equipe de Enfermagem transmite ao paciente durante o pós-operatório, existem muitas outras que surgem da necessidade individual de saber. Nesse processo, a Enfermagem é responsável por dar e receber informações relevantes para possibilitar o enfrentamento de um período com importantes restrições e cuidados como é o pós-operatório de Artroplastia Total de Quadril.

Importantes cuidados específicos pós-operatórios durante o período de internação não foram 
mencionados pelos pacientes, tais como: repouso absoluto no leito até a liberação pelo cirurgião; vigilância e segurança do paciente com estado de consciência alterado; mobilização específica no leito; controle da dor e dor relacionada aos exercícios de reabilitação; avaliação neurovascular; controle de sinais vitais e glicemia; controle das eliminações (risco de constipação induzida por opioide) ${ }^{(6,16-17,19,22-25)}$.

Também foram suprimidas informações sobre: cuidados gerais de Enfermagem; medicamentos específicos; treinamento do paciente e/ou familiar para a administração de medicamentos, realização de curativos, identificação de complicações e cuidados específicos relacionados à cirurgia para após a alta; necessidade de manutenção de drenos, cateteres, etc; oferta e aceitação da dieta; controle de condições que podem afetar a mobilização precoce (dor, náuseas, vômitos, alteração do estado de consciência, etc); prevenção de trombose venosa profunda; cuidados para prevenção de comprometimentos relacionados à síndrome do desuso; realização do curativo; identificação precoce e controle das complicações; atividade e repouso; preparo do paciente e familiar para a alta ${ }^{(6,16-17,19,22-25)}$.

Após a alta, o paciente se encontra mais suscetível às dificuldades e complicações relacionadas à cirurgia, pois não está mais no ambiente controlado por profissionais como no hospital. Esse é um período crítico, em que a maior parte dos problemas relacionados à cirurgia pode resultar na necessidade de outra internação, nova cirurgia, sequelas e até a morte. O preparo para esse período é fundamental para a garantia do sucesso da evolução do paciente porque é muito importante que possam gerir seu cuidado fora do hospital. O sucesso da cirurgia, do tratamento e manutenção da qualidade de vida após a cirurgia pode depender do bom preparo do paciente e de sua família. No entanto, o que percebemos é que um número insuficiente de informações foi mencionado pelos indivíduos pesquisados.

Informações fundamentais e que devem estar consolidadas não só pelo paciente, mas também pela sua família ou cuidadores, além das já mencionadas, são: ambiente domiciliar seguro; limitações e restrições a mobilizações no leito e fora dele; uso de dispositivos e cuidados durante a locomoção; identificação precoce de complicações; cuidados higiênicos; alimentação e hidratação; controle das eliminações fisiológicas; medicamentos específicos, inclusive terapia anticoagulante; acompanhamento e controle das comorbidades; restrições à atividade sexual; restrições à atividade laboral; exercícios de reabilitação no domicílio; retorno às consultas; e controle de risco para quedas ${ }^{(3,6-7,11,16-19,23-24,26)}$.

O conhecimento deficiente, seja por esquecimento por parte do paciente, pelo mesmo não ter dado atenção suficiente no momento em que o profissional de saúde o orientava ou por não terem sido preparado adequadamente, pode levar a complicações evitáveis e causar deterioração do estado de saúde, principalmente no caso dos pacientes idosos. As informações devem ser reconhecidas como parte fundamental do tratamento e responsabilidade de todos os profissionais de saúde envolvidos na assistência.

Cada paciente e seu cuidador deve receber a informação necessária e o reforço dessas informações não só no momento da alta, mas sempre que for possível fazê-la. A forma como essa informação é oferecida deve respeitar a capacidade de compreensão do paciente e do familiar e ser fornecida no momento mais apropriado possível, além do reforço no momento da alta hospitalar. O nível cognitivo, ansiedade, presença de dor, tipo de linguagem utilizada, entre outros, são fatores que podem interferir negativamente na recepção e compreensão da informação e na sua retenção.

Compreender a importância da informação ao paciente cirúrgico denota competência profissional, que é demonstrada pela capacidade do profissional desempenhar as suas funções de forma segura e eficaz, utilizando-se de evidências, com pensamento reflexivo e crítico como parte ativa da equipe multidisciplinar. Isso requer o desenvolvimento de uma prática baseada em evidências, técnicas e conhecimentos específicos e formas de abordar e resolver problemas, sustentados cientificamente. A boa prática tem apontado para o desenvolvimento de diretrizes e protocolos assistenciais multiprofissionais e multidisciplinares que garantam uma abordagem integral ao paciente.

As limitações deste estudo estão relacionadas à reduzida publicação de artigos sobre Enfermagem Ortopédica no Brasil e reduzido acesso a revistas internacionais especializadas, o que gera dificuldades para que a Enfermagem desempenhe suas atividades técnica e cientificamente fundamentadas para este tipo de clientela.

Os resultados deste estudo contribuirão para identificar a situação de compreensão e/ou retenção 
das informações recebidas pelos indivíduos submetidos à Artroplastia Total de Quadril, uma cirurgia de alta complexidade e significativa morbidade e mortalidade na prática hospitalar e após a alta hospitalar. Os dados servirão de subsídios para profissionais de Saúde reconhecerem as fragilidades relacionadas ao preparo do paciente, nas diferentes etapas cirúrgicas, durante o processo de hospitalização e na compreensão e realização dos cuidados domiciliares. A partir do conhecimento produzido, contribuirá para agregar informações que possam ser utilizadas no ensino de profissionais da Enfermagem e também em áreas afins. Espera-se, também, que esta pesquisa sirva como referência para estudos futuros que se relacionem ao tema aqui proposto.

\section{- CONCLUSÃo}

Toda informação fornecida ao paciente, independente do método utilizado, relacionada ao período perioperatório e alta, diminui a ansiedade e o medo gerado pela cirurgia, prepara o paciente para participar ativamente do tratamento, possibilita conviver com as limitações relacionadas à cirurgia e reduz o risco de complicações após a alta.

Este estudo aponta que uma insuficiente quantidade de informações foram lembradas como fornecidas pelos profissionais de saúde, desvelando a grande lacuna que existe no processo de cuidar quando o profissional não está atento às necessidades e à compreensão dos pacientes e ao reforço da aprendizagem, ressaltando a necessidade de reforço das informações para assegurar a sua compreensão e aplicação, de forma a garantir uma evolução mais satisfatória da condição de saúde do paciente.

\section{- REFERÊNCIAS}

1. Rampazo-Lacativa MK, dos Santos AA, Coimbra AMV, D'Elboux MJ. WOMACK and SF-36: instruments for evaluating the health-related quality of life of elderly people with total hip arthroplasty. A descriptive study. Sao Paulo Med. J. [Internet] 2015;133(4) [acesso em 12 dez 2016]. Disponível: http://dx.doi.org/10.1590/15163180.2014.8381508.

2. Andrawis J, Akhavan S, Chan V, Lehil M, Pong D, Bozic KJ. Higher preoperative patient activation associated with better patient-reported outcomes after total joint arthroplasty. Clin Orthop Relat Res. [Internet] 2015;473(8) [acesso em 10 dez 2016]. Disponível: http://dx.doi.org/10.1007/s11999-015-4247-4.

3. Gouveia VR, Mendoza IYQ, Couto BRGM, Ferreira JAG, Paiva EB, Guimarães GL, et al. Profile of hip arthroplasty patients in a teaching hospital. Rev. Col. Bras. Cir. [Internet] 2015;42(2) [acesso em 12 dez 2016]. Disponível: http:// dx.doi.org/10.1590/0100-69912015002007.

4. Camanho G. Editorial - Safe surgery for all. Rev Bras Ortop. [Internet] 2014;49(6) [acesso em 22 nov 2016]. Disponível: http://dx.doi.org/10.1016/j.rbo.2014.09.001.

5. Uesugi Y, Hayashi S, Fujishiro T, Kanzaki N, Nishiyama T. Effectiveness of distance education intervention using video footage in postoperative patients after total hip arthroplasty. Int J Orthop Trauma Nurs. [Internet] 2013;17(2) [acesso em 29 jan 2016]. Disponível: http://dx.doi.org/10.1016/j.ijotn.2013.01.002.

6. Hohler SE. Innovations in total hip arthroplasty. OR Nurse [Internet] 2014;8(1) [acesso em 22 jan 2016]. Disponível: http://dx.doi.org/10.1097/01.ORN.0000433527.64164.98.

7. Darcy AM, Murphy GA, DeSanto-Madeya S. Evaluation of discharge telephone calls following total joint replacement surgery. Orthop Nurs. [Internet] 2014;33(4) [acesso em 05 fev 2016]. Disponível: http://dx.doi. org/10.1097/NOR.0000000000000062.

8. Brent L, Coffey A. Patient's perception of their readiness for discharge following hip fracture surgery. Int J Orthop Trauma Nurs. [Internet] 2013;17(4) [acesso em 29 jan 2016]. Disponível: http://dx.doi.org/10.1016/j. ijotn.2013.01.001.

9. Ministério da Saúde (BR). DATASUS. [Internet] Leitos de internação - Brasil [acesso em 11 mar 2017]. Disponível: http://tabnet.datasus.gov.br/cgi/tabcgi.exe?cnes/cnv/leiintbr.def. 
10. Ministério da Saúde (BR). Conselho Nacional de Saúde. Diretrizes e normas regulamentadoras de pesquisa envolvendo seres humanos. Resolução n. 466, de 12 de dezembro de 2012. Brasília; 2012.

11. Liu XW, Zi Y, Xiang LB, Wang Y. Total hip arthroplasty: a review of advances, advantages and limitations. Int J Clin Exp Med. [Internet] 2015;8(1) [acesso em 06 dez 2016]. Disponível: https://www.ncbi.nlm.nih.gov/pmc/ articles/PMC4358426/.

12. Parsons G, Jester R, Godfrey H. A randomised controlled trial to evaluate the efficacy of a health maintenance clinic intervention for patients undergoing elective primary total hip and knee replacement surgery. Int J Orthop Trauma Nurs. [Internet] 2013;17(4) [acesso em 29 jan 2016]. Disponível: http://dx.doi.org/10.1016/j.ijotn.2013.07.004.

13. Fraval A, Chandrananth J, Chong YM, Coventry LS, Tran P. Internet based patient education improves informed consent for elective orthopaedic surgery: a randomized controlled trial. BMC Musculoskelet Disord. [Internet] 2015;(16) [acesso em 15 out 2016]. Disponível: http://dx.doi.org/10.1186/s12891-015-0466-9.

14. Miller CP, Buerba RA, Leslie MP. Preoperative factors and early complications associated with hemiarthroplasty and total hip arthroplasty for displaced femoral neck fractures. Geriatr Orthop Surg Rehabil. [Internet] 2014;5(2) [acesso em 05 dez 2016]. Disponível: http://dx.doi.org/10.1177/2151458514528951.

15. Vogl M, Wilkesmann R, Lausmann C, Hunger M, Plötz W. The impact of preoperative patient characteristics on health states after total hip replacement and related satisfaction thresholds: a cohort study. Health Qual Life Outcomes. [Internet] 2014;(12) [acesso em 22 nov 2016]. Disponível: http://dx.doi.org/10.1186/s12955-014-0108-1.

16. Doi K, Shimoda R, Gibbons G. Improving pain management in orthopedic surgical patients with opioid tolerance. Nurs Clin North Am. [Internet] 2014;49(3) [acesso em 15 out 2016]. Disponível: http://dx.doi. org/10.1016/j.cnur.2014.05.015.

17. Nanjayan SK, Swamy GN, Yellu S, Yallappa S, Abuzakuk T, Straw R. In-hospital complications following primary total hip and knee arthroplasty in octogenarian and nonagenarian patients. J Orthop Traumatol. [Internet] 2014;15(1) [acesso em 10 dez 2016]. Disponível: http://dx.doi.org/10.1007/s10195-013-0262-y.

18. Soares AB, Silva AM, da Silva GD, de Siqueira ICGL, Pamponet J, da Cruz MP, et al. A assistência de enfermagem ao paciente submetido à artoplastia total de quadril e a importância dos cuidados no período pós operatório. Revista Recien. [Internet] 2013;3(7) [acesso em 28 nov 2016]. Disponível: http://dx.doi.org/10.24276/rrecien2177157X.2013.3.7.11-18.

19. Kapoor A, Chew P, Silliman RA, Hylek EM, Katz JN, Cabral H, Berlowitz D. Venous thromboembolism after joint replacement in older male veterans with comorbidity. J Am Geriatr Soc. [Internet] 2013;61(4) [acesso em 12 dez 2016]. Disponível: http://dx.doi.org/ 10.1111/jgs.12161.

20. Liu SS, Buvanendran A, Rathmell JP, Sawhney M, Bae JJ, Moric M, et al. Predictors for moderate to severe acute postoperative pain after total hip and knee replacement. Int Orthop [Internet] 2012:36(11) [acesso em 21 jul 2016]. Disponível: http://dx.doi.org/10.1007/s00264-012-1623-5.

21. Bentley D, Callum J, Flynn J, Gollish J, Murnaghan J, Lin Y. The benefit of early identification of anemia preoperatively in patients undergoing hip and knee joint arthroplasty. Int J Orthop Trauma Nurs. [Internet] 2014;18(1) [acesso em 29 jan 2016]. Disponível: http://dx.doi.org/10.1016/j.ijotn.2013.07.001.

22. Hoell S, Sander M, Gosheger G, Ahrens H, Dieckmann R, Hauschild G. The minimal invasive direct anterior approach in combination with large heads in total hip arthroplasty - is dislocation still a major issue? a case control study. BMC Musculoskelet Disord. [Internet] 2014;(15) [acesso em 28 jan 2016]. Disponível: http://dx.doi. org/10.1186/1471-2474-15-80.

23. Piolanti N, Andreani L, Parchi PD, Bonicoli E, Niccolai F, Lisanti M. Clinical and radiological results over the medium term of isolated acetabular revision. ScientificWorldJournal. [Internet] 2014;(148592) [acesso em 25 set 2016]. Disponível: http://dx.doi.org/10.1155/2014/148592.

24. Ekpo TE, Berend KR, Morris MJ, Adams JB, Lombardi AV. Partial two-stage exchange for infected total hip arthroplasty: a preliminary report. Clin Orthop Relat Res. [Internet] 2014;472(2) [acesso em 28 nov 2016$].$ Disponível: http://dx.doi.org/10.1007/s11999-013-3168-3.

25. Tayrose G, Newman D, Slover J, Jaffe F, Hunter T, Bosco J 3rd. Rapid mobilization decreases length-of-stay in joint replacement patients. Bull Hosp Jt Dis (2013). [Internet] 2013;71(3) [acesso em 28 set 2016]. Disponível: http:// 
hjdbulletin.org/files/archive/pdfs/81.pdf.

26. Cowie JG, Turnball GS, Ker AM, Breusch SJ. Return to work and sports after total hip replacement. Arch Orthop Trauma Surg. [Internet] 2013;133(5) [acesso em 10 dez 2016]. Disponível: http://dx.doi.org/10.1007/s00402013-1700-2. 